\title{
A New Symbolic Method for Discernibility Matrix in Rough Set
}

\author{
${ }^{1}$ Qianjin Wei and ${ }^{2}$ Tianlong Gu \\ ${ }^{1}$ School of Electronic Engineering, XiDian University, Xi' an, 710071.China \\ ${ }^{2}$ Guangxi Key Laboratory of Trusted Software, Guilin University of Electronic \\ Technology
}

\begin{abstract}
Generating discernibility matrix consumes huge time and space .To solve this problem, A new Binary Discernibility Matrix (BDM) induced from information table is defined, The concept of Binary Conjunction Matrix(BCM) is then introduced, Finally A novel method for discernibility matrix using Zero-Suppressed BDDs (ZBDD) and Ordered binary decision diagrams $(O B D D)$ is proposed in this paper, experiment is carried to compare the storage space of discernibility matrix with that of $Z B D D$ and $O B D D$, results show that the new method has better storage performance and improve the attribute reduction for those information systems with more objects and features.
\end{abstract}

Keywords: rough set; discernibility matrix; Zero-Suppressed BDDs; OBDD

\section{Introduction}

Rough set is one of the methods of data mining. It is an effective tool to deal with inaccurate, inconsistent, incomplete information [1]. The rough set theory has become an attractive field in recent years, and has already been successful applied in many scientific and engineering fields such as machine learning and data mining, it is a key issue in artificial intelligence. Attribute reduction is a research focus in rough set theory. Currently looking for the minimum reduction of an information system is exponential complexity, so it is still the main research to search for fast and efficient algorithms for attribute reduction of rough set theory. In Rough set theory, expresses vagueness, not by means of membership, but employing a boundary region of a set. If the boundary region of a set is empty it means that the set is crisp, otherwise the set is rough (inexact). Nonempty boundary region of a set means that our knowledge about the set is not sufficient to define the set precisely. The indiscernibility relation plays a crucial role in Rough set theory. Due to its importance, the different representations have been developed. Most existing are indiscernibility matrix and indiscernibility graph [5-10].

Ordered Binary Decision Diagrams, graph-based representations of Boolean functions [12], have attracted much attention because they enable us to manipulate Boolean functions efficiently in terms of time and space. There are many cases in which conventional algorith $7 \mathrm{~s}$ can be significantly improved by using BDDs [13-15]. Zero-suppressed BDDs [16] are a new type of BDDs that are adapted for representing sets of combinations. It can manipulate sets of combinations more efficiently than conventional BDDs. especially when dealing with sparse combinations. A Muir, I Düntsch and G Gediga discussed rough set data representation using binary decision diagrams [17], in which, a new information system representation is presented, called BDDIS. Chen Yuming and Miao Duoqian presented searching Algorithm for Attribute Reduction based on Power Graph [5], a new knowledge representation, called power graph, is presented in those paper, therefore, searching algorithms based on power 
graph are also proposed. Visnja Ognjenovic, etc., presents a new form of indiscernibility relation based on graph [17]. In this paper, ZBDD and OBDD are used to represent the family of all equivalence classes of indiscernibility relation; it has been shown how the discernibility matrix can be represented using ZBDD and OBDD.

\section{Indiscernibility Relation in Rough Sets Theory}

The basic concepts, notations and results related to the theory of rough set are briefly reviewed in this section, others can be found in [1-3].

\subsection{Information Systems for Rough Set}

An information system is composed of a 4-tuples as follows:

$\mathrm{I}=\langle\mathrm{U}, \mathrm{Q}, \mathrm{V}, f\rangle$

Where

$\mathrm{U}$ is the closed universe, a finite set of $\mathrm{N}$ objects $\left\{s_{1}, s_{2} \ldots s_{n}\right\}$

$\mathrm{Q}$ is finite attributes $\left\{q_{1}, q_{2} \ldots q_{\mathrm{m}}\right\}$

$\mathrm{V}=\cup_{q \in \mathrm{Q}} \mathrm{V}_{q}$ where $\mathrm{V}_{q}$ is a value of the attribute $q$, called the domain of attribute $q$.

$f: \mathrm{U} \times \mathrm{Q} \rightarrow \mathrm{V}$ is the total decision function called the information function

Such that $f(s, q) \in \mathrm{V} q$ for every $q \in \mathrm{Q}, s \in \mathrm{U}$.Such that $f(s, q)=v$ means that the object $s$ has the value $v$ on attribute $q$.

\subsection{Indiscernibility Relation and Set Approximation}

Let $\mathrm{I}=<\mathrm{U}, \mathrm{Q}, \mathrm{V}, f>$ be an information system, then with any non-empty subset $\mathrm{P} \subseteq \mathrm{Q}$ there is an associated indiscernibility relation denoted by IND (P), it is defined as the following way: two objects $s_{i}$ and $s_{j}$ are indiscernible by the set of attributes $\mathrm{P}$ in Q, if $f\left(s_{i}, q\right)=f\left(s_{j}, q\right)$ for every $q \in \mathrm{P}$, More formally:

$$
\text { IND }(P)=\left\{\left(s_{i}, s_{j}\right) \in U \times U \mid \forall q \in P, f\left(s_{i}, q\right) \neq f\left(s_{j}, q\right)\right\}
$$

Where IND (P) is called the P-indiscernibility relation. If $\left(s_{i}, s_{j}\right) \in \operatorname{IND}(\mathrm{P})$, then objects $s_{i}$ and $s_{j}$ are indiscernible from each other by attributes from P. Obviously IND (P) is an equivalence relation. The family of all equivalence classes of IND (P) will be denoted by U/ IND (P), an equivalence class of IND (P) containing $s$ will be denoted by $[s]_{\operatorname{IND}(\mathrm{P})}$.

$$
\begin{gathered}
U / I N D(P)=\left\{[s]_{I N D(P)} \mid s \in U\right\} \\
{[s]_{I N D(P)}=\{x \in U \mid(s, x) \in I N D(P)\}}
\end{gathered}
$$

Given any subset of attributes $\mathrm{P}$, any concept $\mathrm{X} \subseteq \mathrm{U}$ can be precisely characterized in terms of the two precise sets called the lower and upper approximations. The lower approximation, denoted by $\underline{P X}$, is the set of objects in $\mathrm{U}$, which can be classified with certainty as elements in the concept $\mathrm{X}$ using the set of attributes $\mathrm{P}$, and is defined as follows:

$$
\underline{P} X=\left\{s_{i} \in U \mid\left[s_{i}\right]_{I N D(P)} \subseteq X\right\}
$$

The upper approximation, denoted by $\bar{P} X$, is the set of elements in $\mathrm{U}$ that can be possibly classified as elements in $\mathrm{X}$, and is defined as follows:

$$
\bar{P} X=\left\{s_{i} \in U \mid\left[s_{i}\right]_{I N D(P)} \cap X \neq \Phi\right\}
$$


For any object $s_{i}$ of the lower approximation of X, it is certain that it belongs to X. For any object $s_{\mathrm{i}}$ of the upper approximation of $\mathrm{X}$, we can only say that it may belong to $\mathrm{X}$.

\subsection{Reduct of Rough Set Theory and Independence of Attributes}

Reduct is a fundamental concept of rough set. So-called attribute reduction, it means to delete those dispensable attributes with the same partition of the universe. In another words, the reduct is a minimal subset of attributes, which has the discernible power as using the entire attributes. An important task in rough set based data analysis is computation of the attribute reduction. In order to check whether the set of attributes is independent or not, one checks for every attribute whether its removal increase the number of elementary sets in information system. Given an information table $\mathrm{T}=\langle\mathrm{U}, \mathrm{Q}, \mathrm{V}, f\rangle$, Let $q \in \mathrm{Q}$, attribute $q$ is dispensable in $\mathrm{T}$, if $\operatorname{IND}(\mathrm{U})=\operatorname{IND}(\mathrm{U}-\{q\})$, otherwise $q$ is indispensable in $\mathrm{T}$. A subset $\mathrm{P} \subseteq \mathrm{Q}$ is called a reduct, if $\mathrm{P}$ satisfies the two conditions:

$$
\begin{gathered}
I N D(P)=I N D(Q) \\
\forall q \in P, I N D(P) \neq I N D(P-\{q\})
\end{gathered}
$$

The first condition indicates the sufficiency of the attribute set $\mathrm{P}$, the second condition indicates that each attribute in $\mathrm{P}$ is indispensable. Given an information table, there may exist many reducts, finding all reducts of information system is combinatorial NP-hard computational problem.

\subsection{Definition. Discernibility Matrix}

Given an information system $\mathrm{I}=\langle\mathrm{U}, \mathrm{Q}, \mathrm{V}, f\rangle$, two objects are discernible if their values are different in at least one attribute, the discernibility knowledge of the information system is commonly recorded in a symmetric $|\mathrm{U}| \times|\mathrm{U}|$ matrix $\mathrm{M}_{\mathrm{I}}\left(c_{i j}\left(s_{i}, s_{j}\right)\right)$, called the Discernibility matrix of I. Each element $c_{i j}\left(s_{i}, s_{j}\right)$ for an object pair $\left(s_{i}, s_{j}\right) \in \mathrm{U} \times \mathrm{U}$ is defined as follows:

$$
c_{i j}\left(s_{i}, s_{j}\right)=\left\{\begin{array}{c}
\left\{q \in Q \mid f\left(s_{i}, q\right) \neq f\left(s_{j}, q\right)\right\} \\
\Phi
\end{array}\right.
$$

Since $\mathrm{M}_{\mathrm{I}}\left(c_{i j}\left(s_{i}, s_{j}\right)\right)$ is symmetric and $c_{i i}\left(s_{i}, s_{i}\right)=\varnothing$,For $i=1,2 \ldots \mathrm{m}$, we represent $\mathrm{M}_{\mathrm{I}}\left(c_{i j}\left(s_{i}\right.\right.$, $\left.s_{j}\right)$ ) only by elements in the lower triangle of $\mathrm{M}_{\mathrm{I}}\left(c_{i j}\left(s_{i}, s_{j}\right)\right)$, i.e. the $c_{i j}\left(s_{i}, s_{j}\right)$ is with $1<i<j$ $<\mathrm{m}$.

The physical meaning of the matrix element $c_{\mathrm{ij}}\left(s_{i}, s_{j}\right)$ is that objects $s_{i}$ and $s_{j}$ can be distinguished by any attribute in $c_{i j}\left(s_{i}, s_{j}\right)$, In another words, $c_{i j}\left(s_{i}, s_{j}\right)$ is defined as the set of all attributes which discern object $s_{i}$ and $s_{i}$. The pair $\left(s_{i}, s_{j}\right)$ can be discerned if $c_{i j}\left(s_{i}, s_{j}\right) \neq \varnothing$.

\section{OBDD and Zero-suppressed BDDS}

\subsection{Ordered Binary Decision Diagram}

Given an n-ary Boolean function $f\left(x_{1}, x_{2} \ldots x_{\mathrm{n}}\right)$, an ordered binary decision diagram (OBDD) is a finite directed acyclic graph with one root, $\mathrm{n}+1$ levels, and exactly two branches at each non-terminal node [12]. One of these is the 0 case, denoted by low $(x)$ and drawn as a dashed line, the other the 1 case, denoted by high $(x)$ and drawn as a solid line. The levels are determined by the fixed ordering of the variables $x_{i}<x_{j}<\ldots<x_{k}$. Each traversal through the tree corresponds to an assignment to the variables, and the nodes at level $n+1$ give the evaluation of $f$ corresponding through this traversal. 
For example, Figure 1 shows a binary decision tree (the reduction rules are not applied) for the function $(x+y) \cdot z$

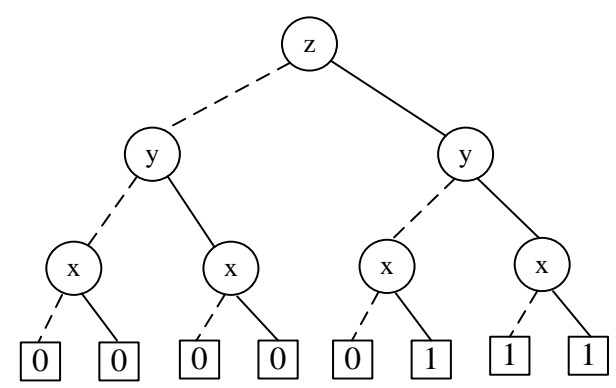

Figure 1. Binary Decision Tree for the Function $(x+y) \cdot z$

The following reduction rules do not change the value of the function:

1) Delete redundant terminal nodes and only one of them is reserved, one terminal label for 0 and one terminal label for 1 , redirect all lines from level $\mathrm{n}$ to the respective node.

2) For non-terminal nodes $u$ and $v$, if $\mathrm{u}$ and $\mathrm{v}$ are on the same level and low $(u)=$ low $(v)$, high $(u)=$ high $(v)$, then delete one of them, and all entry edges of the deleted node should point to the reserved node.

3) For non-terminal node $\mathrm{u}$, if low $(u)=$ high $(u)$, then delete $\mathrm{u}$, and all the entry edges of $u$ should point to low $(u)$.

The binary decision tree of the Figure 1 can be transformed into an ordered binary decision diagram (OBDD) by maximally reducing it according to the above reduction rules. The advantage of an OBDD is that it is unique for a particular function and variable order. This property makes it useful in functional equivalence checking and other operations like functional technology mapping. A path from the root node to the 1-terminal represents a variable assignment for which the represented Boolean function is true. Figure 2 shows an OBDD for the Boolean function $f(x, y, z)=(x+y) . z$. We trace the path (1) $\rightarrow(2) \rightarrow(3) \rightarrow(4)$, and reach the terminal node 1 . Thus, the value of Boolean function $f(x, y, z)=(x+y)$. $z$ of variable assignment $(0,1,0)$ is 1 .

OBDD have some interesting properties. They provide compact representations of Boolean expressions, and there are efficient algorithms for performing all kinds of logical operations on OBDD. They are all based on the crucial fact that for any Boolean function $f$ there is exactly one OBDD representing it. This means, in particular, that there is exactly one OBDD for the constant true function. Hence, it is possible to test in constant time whether an OBDD is constantly true or false.

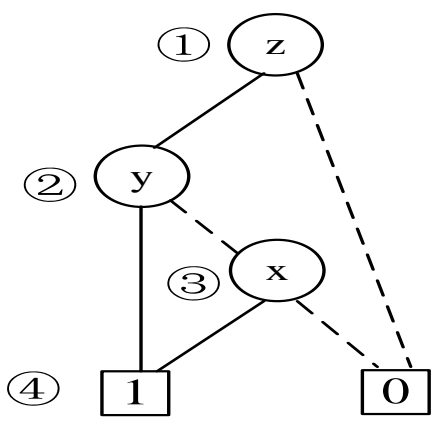

Figure 2. OBDD for $(x+y) \cdot z$. 


\subsection{Combination Sets}

Sets of combinations often appear in solving combinatorial problems. The representation and manipulation of sets of combinations are important techniques for many applications. A combination on $n$ objects can be represented by an $n$ bit binary vector, $\left(x_{1} x_{2} \ldots x_{\mathrm{n}}\right)$, where each bit, $x_{k} \in\{0,1\}$, expresses whether the corresponding object is included in the combination or not. A set of combinations can be represented by a set of the $n$ bit binary vectors. In other words, combination sets is a subset of the power set of $\mathrm{n}$ objects. We can represent a combination set with a Boolean function by using n-input variables for each bit of the vector, If we choose any one combination vector, a Boolean function determines whether the combination is included in the set of combinations. Such Boolean functions are called characteristic functions. For example, given three elements $\{x, y, z\}$, consider the set of subsets $\{\{x, y\},\{x, z\},\{z\}\}$.If we associate each element with a binary variable having the same name, the characteristic function of the set of subsets is $f=x y z^{\prime}+x y^{\prime} z+x^{\prime} y^{\prime} z$. The first minterm corresponds to the subset $\{x, y\}$, and so on. The operations of sets, such as union, intersection and difference, can be executed by logic operations on characteristic functions.

Once the characteristic function is constructed, it can be represented using an OBDD or ZBDD. The two representations of the set of subsets $\{\{x, y\},\{x, z\},\{z\}\}$ are given in Figure 4. In both diagrams, there are three paths from the root node to the1-terminal node, which correspond to the subsets $\{x, y\},\{x, z\}$, and $\{z\}$.By using OBDD for characteristic functions, we can manipulate sets of combinations efficiently. Due to the effect of node sharing, OBDD compactly represent sets of huge numbers of combinations. Despite the efficiency of OBDD, there is one inconvenience that the forms of OBDD depend on the input domains when representing sets of combinations. This inconvenience comes from the difference in the model on default variables. In combination sets, default variables are regarded as zero when the characteristic function is true, since the irrelevant objects never appear in any combination. Unfortunately, such variables cannot be suppressed in the OBDD representation, and many useless nodes are generated when we manipulate sparse combinations. This is the reason why we need another type of OBDD for manipulating sets of combinations.

\subsection{Zero-suppressed BDDs}

Zero-suppressed BDDs are a new type of BDD adapted for representing sets of combinations [16]. This data structure is more efficient and simpler than usual BDDs when we manipulate sets in combinatorial problems. They are based on the following reduction rules:

PD-deletion rule: Delete all nodes whose 1-edge points to the 0 -terminal node, and then connect the edges to the other subgraph directly, as shown in Figure 3.

Merging rule: Share all equivalent subgraphs in the same manner as with conventional BDDs.

Notice that we do not delete the nodes whose two edges point to the same node. The zerosuppressed deletion rule is asymmetric for the two edges, as we do not delete the nodes whose 0 -edge points to a 0 -terminal node. 


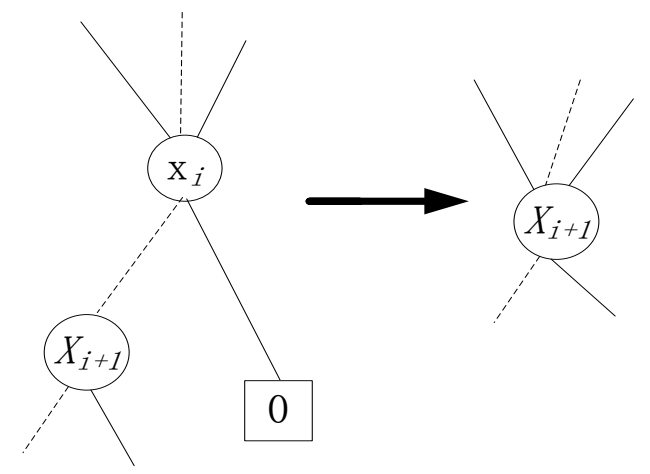

Figure 3. PD-deletion Rule

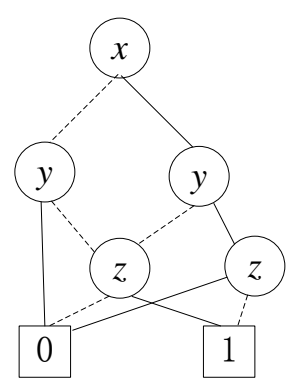

(a)

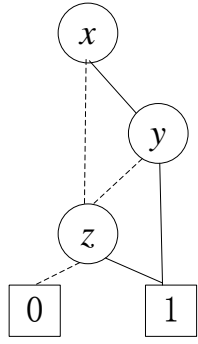

(b)

Figure 4. OBDD and ZBDD for the Set of Subsets $\{\{x, y\},\{x, z\},\{z\}\}$

Figure 4(b) shows the ZBDDs for the same sets of combinations shown in Figure 4 (a). The form of ZBDDs is independent of the input domain. The ZBDDs node deletion rule automatically suppresses the variables which never appear in any combination. This feature is important when we manipulate sparse combination.

\section{Ordered Binary Decision Diagram Discernibility Matrix}

In this section, a new method to represents indiscernibility within an information system is put forward, called Ordered Binary Decision Diagram Discernibility Matrix (OBDDDM). Specific steps of the method that construct OBDDDM of an system are as follows:

Step1: Transform discernibility matrix $\mathrm{M}_{\mathrm{I}}\left(c_{i j}\left(s_{i}, s_{j}\right)\right)$ into Binary Discernibility Matrix (BDM), which is an important concept for indiscernibility relation.

Step2:According to the meaning of the discernibility matrix,BDM can be simplified: delete those rows which all elements are 1 and rows which are unique 1(as the attribute core);delete duplicate rows, the modified concept of indiscernibility called Binary Conjunction Matrix $(\mathrm{BCM})$ is introduced.

Step3: According to BCM, encode attribute, thus OBDDDM corresponding to information system can be derived.

An information system is illustrated in Table 1, which has five objects with $\mathrm{U}=\left\{s_{1}, s_{2}, s_{3}, s_{4}, s_{5}\right\}$ and five attributes with $\mathrm{Q}=\{a, b, c, d, e\}$, with rows representing objects and columns representing attributes. 
Table 1. An Information System

\begin{tabular}{cccccc}
\hline \multirow{2}{*}{$\mathrm{U}$} & \multicolumn{5}{c}{$\mathrm{Q}$} \\
\cline { 2 - 6 } & $a$ & $b$ & $c$ & $d$ & $e$ \\
\hline$s_{1}$ & 1 & 0 & 2 & 1 & 0 \\
$s_{2}$ & 0 & 0 & 1 & 2 & 1 \\
$s_{3}$ & 2 & 0 & 2 & 1 & 0 \\
$s_{4}$ & 0 & 0 & 2 & 2 & 2 \\
$s_{5}$ & 1 & 1 & 2 & 1 & 0 \\
\hline
\end{tabular}

Table 2. Discernibility Matrix for the Information System in Table 1

\begin{tabular}{cccccc}
\hline $\mathrm{U}$ & $s_{1}$ & $s_{2}$ & $s_{3}$ & $s_{4}$ & $s_{5}$ \\
\hline$s_{1}$ & & & & & \\
$s_{2}$ & $\{a, c, d, e\}$ & & & & \\
$s_{3}$ & $\{a\}$ & $\{a, c, d, e\}$ & & & \\
$s_{4}$ & $\{a, d, e\}$ & $\{c, e\}$ & $\{a, d, e\}$ & & \\
$s_{5}$ & $\{b\}$ & $\{\mathrm{a}, b, c, d, e\}$ & $\{a, b\}$ & $\{a, b, d, e\}$ & \\
\hline
\end{tabular}

The discernibility matrix of Table 1 is shown Table 2 , for the underlined object pair $\left(s_{1}, s_{2}\right)$, the entry $\{a, c, d, e\}$ indicates that attribute a, c, $\mathrm{d}$ or e discerns the two objects.

According to Table 1, we have the following partitions defined by attribute sets $\{a\},\{b\}$, $\{c\}$ and $\{a, b\}$ :

$\mathrm{U} / \mathrm{IND}(\{a\})=\left\{\left\{s_{1}, s_{5}\right\},\left\{s_{2}, s_{4}\right\},\left\{s_{3}\right\}\right\}$

$\mathrm{U} / \mathrm{IND}(\{b\})=\left\{\left\{s_{1}, s_{2}, s_{3}, s_{4}\right\},\left\{s_{5}\right\}\right\}$

$\mathrm{U} / \mathrm{IND}(\{c\})=\left\{\left\{s_{1}, s_{3}, s_{4}, s_{5}\right\},\left\{s_{2}\right\}\right\}$

If we have the set $\{\mathrm{a}, \mathrm{b}\}$ we will have:

$\mathrm{U} / \mathrm{IND}(\{a, b\})=\left\{\left\{s_{1}\right\},\left\{s_{2}, s_{4}\right\},\left\{s_{3}\right\},\left\{s_{5}\right\}\right\}$

\subsection{BDM and BCM}

In the first step, an improved definition of a binary matrix to represent discernibility between pairs of objects, called Binary Discernibility Matrix, Suppose object pair $\left(s_{i}, s_{j}\right)$ $\in \mathrm{U} \times \mathrm{U}$, the row of Binary Discernibility Matrix is n-dimensional binary vector. In which, the kth component $q_{k}\left(s_{i}, s_{j}\right)=0(k=1,2 \ldots n)$ indicates that $s_{i}$ and $s_{j}$ is indistinguishable on the attribute $q_{k}$, in another words, the object $s_{i}$ has the same value $v$ on attribute $k$ with the object $s_{j}$. The kth component $q_{k}\left(s_{i}, s_{j}\right)=1(k=1,2 \ldots n)$ indicates that $s_{i}$ and $s_{j}$ is discernible on the attribute $q_{k}$, in another words, the object $s_{i}$ has the different value $v$ on attribute $q_{k}$ with the object $s_{j}$. For example, Table 3 shows the Binary Discernibility Matrix for the information system in Table 1, which consists of five objects $\mathrm{U}=\left\{s_{1}, s_{2}, s_{3}, s_{4}, s_{5}\right\}$, five attributes $\mathrm{Q}=\{a, b, c, d, e\}$ for example, the first line is [10111], it means that the two objects $s_{i}$ is distinguishable on the attribute sets $\{a, c, d, e\}$ with the object $s_{j}$.

Table 3. Binary Discernibility Matrix for Discernibility matrix in Table 2

\begin{tabular}{ccccc}
\hline$a$ & $b$ & $c$ & $d$ & $e$ \\
\hline 1 & 0 & 1 & 1 & 1 \\
1 & 0 & 0 & 0 & 0 \\
1 & 0 & 0 & 1 & 1 \\
0 & 1 & 0 & 0 & 0 \\
1 & 0 & 1 & 1 & 1 \\
0 & 0 & 1 & 0 & 1 \\
1 & 1 & 1 & 1 & 1 \\
1 & 0 & 0 & 1 & 1 \\
1 & 1 & 0 & 0 & 0 \\
\hline
\end{tabular}


Table 4. Binary Conjunction Matrix for Discernibility matrix in Table 2

\begin{tabular}{ccccc}
\hline$a$ & $b$ & $c$ & $d$ & $e$ \\
\hline 1 & 0 & 1 & 1 & 1 \\
1 & 0 & 0 & 1 & 1 \\
0 & 0 & 1 & 0 & 1 \\
1 & 1 & 0 & 0 & 0 \\
\hline
\end{tabular}

\subsection{Order Binary Decision Diagram Discernibility Matrix}

Suppose a Binary Conjunction Matrix for Discernibility matrix with $|\mathrm{Q}|$ attributes, we can encode the attributes with a $|\mathrm{Q}|$-dimensional binary vector $\left[x_{0} x_{1} \ldots x_{u-1}\right]$, Therefore, these five attributes of the information system in Table 1 can be represented by 5-dimensional binary vector $\left[x_{0} x_{1} x_{2} x_{3} x_{4}\right]$, let $a=[1000], b=[01000], c=[00100], d=[00010], e=[00001]$. From the information system of Table 1 the OBDDDM of Figure 5 is derived. The path reach the terminal node 0 is omitted. Attribute set $\{a, b, c, d, e\}$ is encoded with the variables $\left[x_{0} x_{1} x_{2} x_{3} x_{4}\right]$. For example, By Figure 5, the rightmost path $x_{0}{ }^{\prime} x_{1}{ }^{\prime} x_{2} x_{3}{ }^{\prime} x_{4}$ from the root node to the 1-terminal indicates two objects $s_{i}$ and $s_{j}$ can be discernibility on the attribute $\{c, e\}$, that is, object $s_{i}$ has the different value on attribute $\{c, e\}$ with the object $s_{j}$.

\section{ZBDDs Discernibility Matrix}

It has been shown how the indiscernibility relations can be obtained by discernibility matrix. The application of the discernibility matrix enables the partitioning or the universe of objects represented by their attributes. We can construct the set for it. For example, the set for the information system in Table 1 is $\{\{a, c, d, e\},\{a\},\{a$, $d, e\},\{b\},\{a, c, d, e\},\{c, e\},\{a, b, c, d, e\},\{a, d, e\},\{a, b\}\}$.

On the foundation of research above, the crucial problem is to create unique ZBDDs of indiscernibility relation for all subsets of attributes Q, this can be done by the following way. We can encode the attributes with $\mathrm{m}$ - bit binary vector $\left[\begin{array}{lll}q_{1} & q_{2} \ldots & q_{\mathrm{m}}\end{array}\right]$, where each bit, $q_{k} \in\{0$, 1 , expresses whether or not the attribute $q_{k}$ is included in the combination. Consider Q, the equivalence class of the equivalence relation of IND (Q) can be represented by $\mathrm{m}$ bit binary vector $\left[\begin{array}{lll}q_{1} & q_{2} \ldots & q_{\mathrm{m}}\end{array}\right]$. First ,we can reduction the set $\{\{\{a, c, d, e\},\{a\},\{a$, $d, e\},\{b\},\{a, c, d, e\},\{c, e\},\{a, b, c, d, e\},\{a, d, e\},\{a, b\}\}$.

Consider $\{a, b, c, d, e\}$, it shows the two objects different in all attributes, so it can be deleted from set. For those set is only one attribute, which must exist in the collection of each reduction, here we can delete the set $\{a\}$ and $\{b\}$. Duplicate entries can be deleted Keep only one $\{a, d, e\}$, At last ,we refer the set to $\{\{a, c, d, e\},\{a, d, e\},\{c, e\},\{a, b\}\}$,it can be represented using a ZBDDs,called ZBDDDM.The ZBDDDM of Discernibility Matrix in Table 2 is shown in Figure 6. 


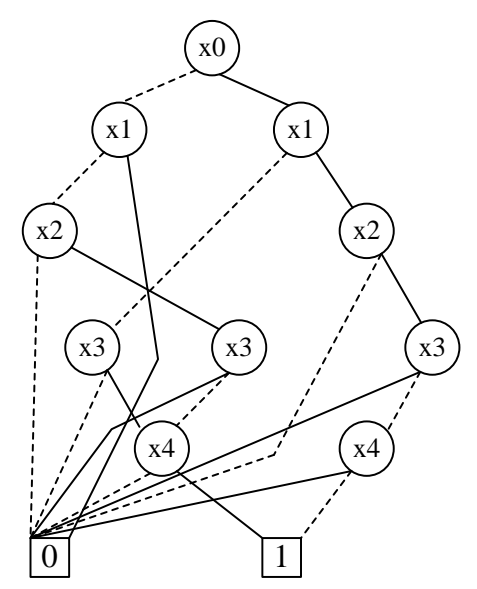

Figure 5. OBDDDM of Discernibility Matrix in Table 2

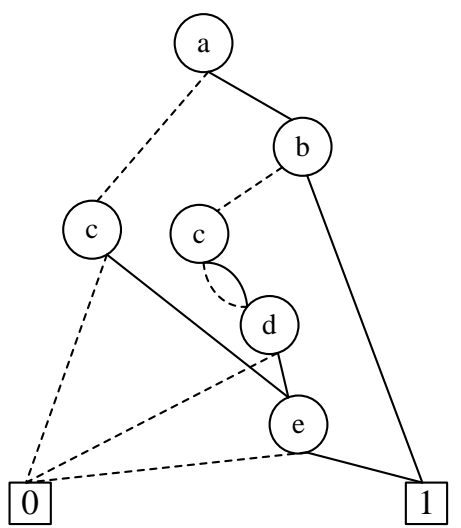

Figure 6. ZBDDDM of Discernibility Matrix in Table 2

\section{Experiments and Conclusions}

\subsection{Experimental Results}

Through the experimental results, The Storage efficiency used in the proposed method with the discernibility matrix. Data sets are random generated by random number generator. Table 5. List the storage efficiency of the Discernibility matrix, OBDDDM, ZBDDDM .As seen in Table 5. The proposed methods is more efficient than the traditional discernibility matrix. Especially on complex information system with more objects and attributes. Because all identical nodes are shared and all redundant tests are eliminated, OBDD and ZBDD have some very convenient properties; therefore, the storage of the OBDDDM and ZBDDDM required is less than that of discernibility matrix. For example, the data of the second group in Table 5, the storage required by discernibility matrix is 1.97 times the storage required by OBDD and the data or the $9^{\text {th }}$ group achieves 3.8 times. Experimental results show that the OBDDDM and ZBDDDM has better storage performance and can improve the attribute reduction of complex information system with more objects and attributes.

\subsection{Conclusion}

Attribute reduction is fundamental in rough set theory. The knowledge representations for discernibility matrix in rough set have been investigated in the paper. The method called 
OBDDDM and ZBDDDM is introduced, for a given information system, as seen from the experiments, the new proposed method is more efficient than that of discernibility matrix. In further research, optimization symbolic algorithms for attribute reduction based on this method will be developed.

Table 5. Experimental Results

\begin{tabular}{|c|c|c|c|c|c|}
\hline \multirow{2}{*}{ Dataset } & \multirow{2}{*}{ object } & \multirow{2}{*}{ Attribute } & \multicolumn{3}{|c|}{ Size(KB) } \\
\cline { 4 - 6 } & & & Discernibility matrix & OBDDDM & ZBDDDM \\
\hline 1 & 7 & 5 & 0.19 & 0.20 & 0.18 \\
\hline 2 & 32 & 57 & 139.69 & 70.75 & 68.91 \\
\hline 3 & 178 & 14 & 265.89 & 133.61 & 140.21 \\
\hline 4 & 200 & 5 & 129.21 & 64.28 & 63.12 \\
\hline 5 & 200 & 25 & 520.56 & 230.13 & 218.29 \\
\hline 6 & 435 & 17 & 560.32 & 239.56 & 249.01 \\
\hline 7 & 1000 & 11 & 785.36 & 280.41 & 300.96 \\
\hline 8 & 2000 & 61 & 1062.51 & 342.75 & 319.15 \\
\hline 9 & 10000 & 50 & 3215.36 & 846.16 & 890.06 \\
\hline
\end{tabular}

\section{Acknowledgements}

This work has been supported by National Natural Science Foundation of China (No.61262030) and Science Foundation of Guanxi Key Laboratory of Trusted Software (No. kx201314).

\section{References}

[1] Z .Pawlak, "Rough sets", International Journal of Information and Computer Science, vol. 11, no. 5, (1982).

[2] A .Skowron and Rauszer, "The discernibility matrices and functions in information systems", in: R. Slowinski (Ed.), Intelligent Decision Support-handbook of Applications and Advances of the Rough Sets Theory, Kluwer Academic Publishers, Dordrecht, (1992).

[3] Z. Pawlak, "Rough set approach to multi-attribute decision analysis", European Journal of Operational Research, vol. 72, no. 3, (1994).

[4] Z. Pawlak and A. Skowron, "Rudiments of rough set", Information Science, vol. 177, no. 1, (2007).

[5] S. K. M. Wong and W. Ziarko, "On optimal decision rules in decision tables. Bulletin of Polish Academy of Sciences", vol. 33, (1985).

[6] Y. M. Chen, D. Q. Miao, R. Z. Wang and K. S. A. Wu, "rough set approach to feature selection based on power set tree", Knowledge based Systems, vol. 24, (2011).

[7] Y. Y. Yao and Y. Zhao, "Discernibility Matrix Simplification for constructing attribute ruducts", Information Sciences, vol. 179, no. 7, (2009).

[8] K. L. Thangave and A. Pethalakshmi, "Dimensionality reduction based on rough set theory: A review", Applied Soft Computing, vol. 9, (2009).

[9] X. H. Hu and N. Cercone, "Learning in relational databases: A rough set approach", Computational Intelligence, vol. 11, no. 2, (1995).

[10] D. Y. Ye and Z. J. Chen, "A new discernibility matrix and the computation of a core", Acta Electronica Sinica, vol. 30, no. 7, (2002).

[11] G. Y. Wang, "Calculation methods for core attributes of decision table", Chinese Journal of Computer, vo. 26, no. 5, (2003).

[12] Q. Liu, "Rough sets and Rough Reasoning”, Science Press, Beijing, (2001).

[13] R E. Bryant, "Graph-based algorithms for Boolean function manipulation”, IEEE Transactions on Computers, vol. 35, no. 8, (1985).

[14] T. L. Gu, Z. B. Xu and Z. F. Yang, "Symbolic OBDD representations for mechanical assembly sequences", Computer-Aided Design, vol. 40, (2008).

[15] T. L. Gu and Z. B. Xu, "Ordered binary decision diagram and it s application”, Science Press, Beijing, (2009).

[16] Y. Matsunaga and M. Fujita, "Multi-level Logic Optimization Using Binar y Decision Diagrams", IEEE International Conference on Computer Aided Design'89, (1989) November, Santa, Clara.

[17] S. Minato, "Zero-suppressed BDDS for set manipulation in combinatorial problems", Proceeding of 30th ACM/IEEE Design automation Conference. 
[18] A, Muir, I. Düntsch and G. Gediga, "Rough set data representation using binary decision diagrams", Computational Sciences, vol. 98, no. 1, (2004).

[19] V. Ognjenovic, V. Brtka, M. Jovanovic, E. Brtka and I. Berkovic, "The Representation of Indiscerniblity Relation by Graph", Proceeding of 9th IEEE International Symposium on Intelligent systems and Informatics.

\section{Authors}

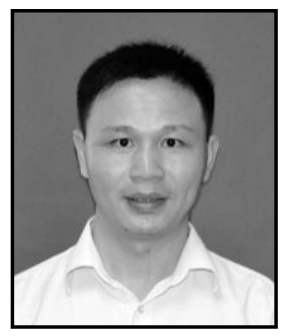

Qianjin Wei, he was born in Hunan, China, on March 1978. He received the Bachelor Degree from Harbin Institute of Technology in 2000, received Master Degree from Kunming University of Science and Technology in 2003. He is currently a Ph.D. candidate in School of Electronic Engineering at XiDian University. His main research interests include formal method, rough set, and knowledge engineering.

Tianlong Gu, he was born in Shanxi, China, on 1st October 1964. He received the Bachelor Degree from Taiyuan University of Technology in 1984, received Master Degree from Xidian University in 1986 and received his Ph.D. degree from Zhejiang University in 1996. From 1998 to 2002, he was a postdoctoral research fellow and visiting professor within Murdoch University and Curtin University of Technology, Australia. He has published more than 130 papers, and authored 6 books. His main research interests include formal method, knowledge engineering and mobile computing. He is a full professor in school of computer science \& engineering at Guilin University of Electronic Technology, Guilin, China, and Ph.D. supervisor in school of computer science \& technology at Xidian University, Xian, China. 
International Journal of Hybrid Information Technology Vol. 7, No. 6 (2014) 